Article

\title{
Island DC Microgrid Hierarchical Coordinated Multi-Mode Control Strategy
}

\author{
Zhongbin Zhao ${ }^{1}$, Jing Zhang ${ }^{1, * \mathbb{D}}$, Yu He ${ }^{1, *}$ and Ying Zhang ${ }^{2}$ \\ 1 School of Electrical Engineering, Guizhou University, Guiyang 550025, China \\ 2 Guizhou Power Grid Company, Guiyang 550001, China \\ * Correspondence: zhangjing@gzu.edu.cn (J.Z.); yhe7@gzu.edu.cn (Y.H.)
}

Received: 2 July 2019; Accepted: 1 August 2019; Published: 5 August 2019

\begin{abstract}
As renewable energy sources connecting to power systems continue to improve and new-type loads, such as electric vehicles, grow rapidly, direct current (DC) microgrids are attracting great attention in distribution networks. In order to satisfy the voltage stability requirements of island DC microgrids, the problem of inaccurate load power dispatch caused by line resistance must be solved and the defects of centralized communication and control must be overcome. A hierarchical, coordinated, multiple-mode control strategy based on the switch of different operation modes is proposed in this paper and a three-layer control structure is designed for the control strategy. Based on conventional droop control, a current-sharing layer and a multi-mode switching layer are used to ensure the stable operation of the DC microgrid. Accurate load power dispatch is satisfied using a difference discrete consensus algorithm. Furthermore, virtual bus voltage information is applied to guarantee smooth switching between various modes, which safeguards voltage stability. Simulation verification is carried out for the proposed control strategy by power systems computer aided design/electromagnetic transients including DC (PSCAD/EMTDC). The results indicate that the proposed control strategy guarantees the voltage stability of island DC microgrids and accurate load power dispatch under different operation modes.
\end{abstract}

Keywords: direct current (DC) microgrid; multi-mode smooth switch; droop control; difference discrete consensus algorithm; hierarchical coordinated control

\section{Introduction}

The microgrid has played an important role in providing reliable access to distributed generation in recent years [1-3]. There are three types of microgrid, namely the DC microgrid, the alternating current $(\mathrm{AC})$ microgrid, and the alternating current/direct current (AC/DC) hybrid microgrid, defined in terms of the difference in bus voltage forms [4]. In comparison with AC microgrids, the advantages of DC microgrids, such as efficient energy conversion, low loss and without conversion of multi-level converters and without considering reactive power loss and frequency, attract constant attention $[5,6]$. Meanwhile, a large number of flexible technologies and pieces of equipment based on power electronics are applied to the distribution network. It is crucial to understand the coordinated control of each unit of a DC microgrid in order to recognize reasonable load power dispatch and stable voltage $[7,8]$.

The coordinated control strategies of DC microgrids mainly include centralized control, distributed control, and decentralized control. Under the centralized control strategy $[9,10]$, the power balance of various units in the DC microgrid is realized with the help of central controller. A bidirectional and high-bandwidth communication line is required to establish a connection between the central controller and each unit. The microgrid is highly dependent on the central controller and this may lead to communication block. Decentralized control [11,12] has no communication requirement and each agent completes its control objective independently. However, owing to the lack of exchange of 
necessary information, the overall control objective cannot be completed. The maximum advantage of distributed control [13-15] is that it has no need to rely on the central controller to achieve point-to-point information exchange. With a sparse communication network, each unit can complete control in terms of its own information and that of the adjacent unit. This may overcome the disadvantage of centralized control, while also achieving the objective of overall control. Even when some units break down or the communication structure changes, stable operation can still be guaranteed. In a parallel-operated microgrid, current-sharing is achieved by means of distributed droop control, so as to meet the plug-and-play requirement of system. However, considering that system load dispatch precision is affected by line parameters, power dispatch errors may occur under the traditional droop control strategy and, consequently, system stability may be affected [16,17].

To understand power coordination control of each unit in a system and guarantee bus voltage stability, a DC microgrid real-time power coordinated strategy was proposed [18]. The system was designed in multi-mode operation in terms of common bus voltage fluctuation. In this way, the DC microgrid can be operated stably. However, the power flow may cause an inequality of bus voltage at different nodes due to the influence of line resistance. In addition, the influence of line resistance on load power dispatch was not considered, which may have caused an overload of some energy storage units and even system breakdown. As a major control mode of DC microgrid operation, droop control is usually adopted in parallel connection with the multi-converter in a DC microgrid. Researchers [19] put forward a coordinated control strategy for an autonomous DC microgrid with a dynamic load power dispatch. The microgrid coordinately operated under different working modes in terms of adaptive droop control. Nevertheless, it failed to consider the influence of factors, such as the inconformity of outlet parameters and the internal resistance of the converter on load power dispatch. Furthermore, the precision of the mode switch may have been reduced due to line resistance. Researchers [20] put forward a decentralized control method for DC microgrids with improved current-sharing accuracy. However, the charge-discharge capacity of the energy storage unit was not considered, which can lead to a power imbalance in the system and consequent system breakdown. Researchers [21] proposed an accurate power dispatch and zero steady-state error voltage-control strategy based on adaptive droop characteristics. This overcame the defects of centralized communication and the system disorders caused by communication breakdown, achieving bus voltage stability and accurate load power sharing. However, the distributed generation capacity may not have been fully utilized and the influence of bus piecewise resistance on load power dispatch was not considered.

In order to solve the problems mentioned above, a hierarchical, coordinated control strategy for an island DC microgrid based on multi-mode smooth switch is proposed in this paper. It aims at fully utilizing distributed generation and guaranteeing stable operation of the system. A three-layered control structure is designed. Based on conventional droop control, a current-sharing layer control for the purpose of accurate load power dispatch and a multi-mode smooth switch layer control aiming at guaranteeing voltage stability are proposed. By means of real-time monitoring of virtual bus voltage information, the smooth switch among various modes of each unit is completed. At the same time, each unit conducts information among adjacent units under different operation modes. Utilizing a difference discrete consensus algorithm (DDCA), accurate load power dispatch and stable operation of the DC microgrid are guaranteed. This paper introduces the DC microgrid mode in Section 2 and puts forward island DC microgrid multi-mode. Furthermore, it presents the hierarchical, coordinated control strategy based on multi-mode smooth switch. Finally, it utilizes PSCAD/EMTDC to verify the effectiveness of the proposed control strategy.

\section{DC Microgrid Structure}

The structure of the island DC microgrid is shown in Figure 1. The island DC microgrid contains photovoltaic arrays, energy storage units, common DC loads, and converters. 


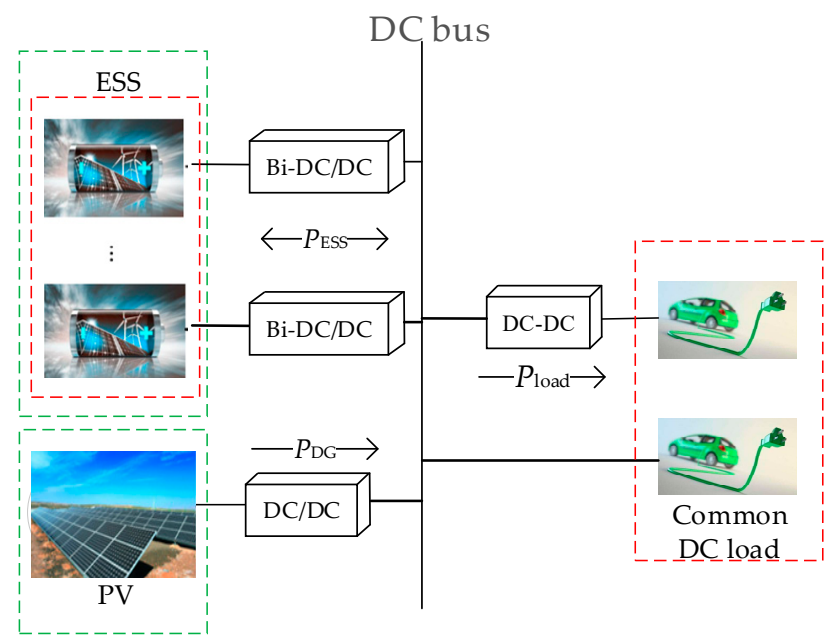

Figure 1. Structure of the island DC microgrid.

(1) Photovoltaic array (PV): Integrated into the DC microgrid bus via a one-way DC/DC converter. PV usually adopts the maximum power point tracking (MPPT) mode to fully utilize PV energy.

(2) Energy storage system (ESS): The energy storage system utilizes multiple group battery energy storage (BES) to meet generation and load fluctuation and integrates this with the DC microgrid bus via a two-way DC/DC converter to adjust the power balance of the system.

(3) Common DC load: The common DC load can be integrated into the DC microgrid bus. In addition, it is possible to carry out constant voltage control for important loads through a double closed-loop DC/DC control system. In this way, the voltage fluctuation is reduced and power-supply stability is improved.

(4) Direct current/direct current converter (DC/DC): This is utilized to achieve connections between various generation units in the grid and important loads, which facilitates power exchange of various units.

\section{Multi-Mode of Island DC Microgrid}

The island DC microgrid operation is divided into three modes in this paper, as indicated in Figure 2. It aims at fully utilizing photovoltaic energy and safeguarding the power balance of the PV-BES load in the island DC microgrid.

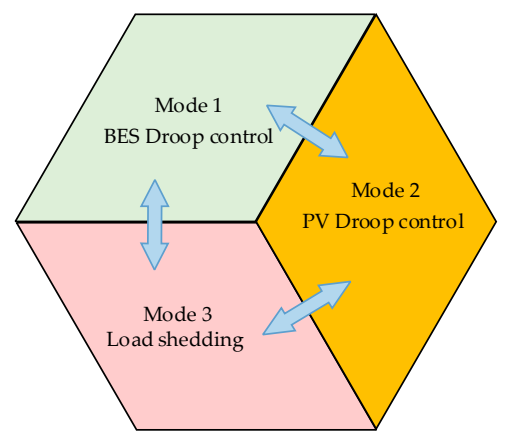

Figure 2. Multi-mode of DC microgrid. 
(1) Mode 1: PV utilizes MPPT control to fully use the photovoltaic energy and maintain a power balance in the system with the help of BES. The power relationship between photovoltaic energy and storage load in the system at this point is indicated by the following formula:

$$
\sum_{i=1}^{N} P_{\mathrm{BES} . i}+\sum_{j=1}^{M} P_{\mathrm{PV} . j}^{\mathrm{MPPT}}=P_{\text {load }}^{\text {sum }}+P_{\mathrm{L}},
$$

where $P_{\mathrm{BES} . i}$ refers to the developed power of $\mathrm{BES} i, P_{\mathrm{PV} . j}^{\mathrm{MPPT}}$ indicates the maximum power point tracking power of PV $j, P_{\text {load }}^{\text {sum }}$ represents the common DC overall load of the system, and $P_{\mathrm{L}}$ indicates the power consumed by line resistance.

(2) Mode 2: In case of the power required by the system exceeding BES safe output capacity with excess power, the system power balance is guaranteed by reducing PV output power. At this point, the system power satisfies the following formula:

$$
\sum_{i=1}^{N} P_{\mathrm{BES} . i}^{\max }+\sum_{j=1}^{M} P_{\mathrm{PV} . j}=P_{\mathrm{load}}^{\text {sum }}+P_{\mathrm{L}}
$$

where $P_{\mathrm{BES} . i}^{\max }$ indicates the maximum safe output power of storage $i$.

(3) Mode 3: When the bus voltage plummets due to the power vacancy of the system, it is necessary to carry out load-shedding control to guarantee the power supply quality of important loads. At this time, the system power satisfies the following formula:

$$
\sum_{i=1}^{N} P_{\mathrm{BES} . i}^{\max }+\sum_{j=1}^{M} P_{\mathrm{PV} . j}^{\mathrm{MPPT}}=P_{\text {load }}+P_{\mathrm{L}},
$$

where $P_{\text {load }}$ indicates the residual load after load shedding.

\section{Hierarchical, Coordinated Control Strategy Based on Multi-Mode Smooth Switch of an Island DC Microgrid}

When the microgrid is affected by the line resistance, traditional droop control cannot achieve precise load power dispatch. In addition, considering that line resistance is hard to measure precisely and the line current is constantly changing, it is difficult to find the precise value of $P_{\mathrm{L}}$. Therefore, it is hard to achieve accurate mode switching in terms of system power fluctuation, as mentioned in Section 3. In order to solve the problem, a hierarchal, coordinated multi-mode control strategy for island DC microgrids is put forward in this paper, as indicated in Figure 3. This strategy fully considers the maximum charge-discharge capacity of BES, as well as the fluctuation of PV and load. In Section 4.1, the influence of line resistance on precise load power dispatch is analyzed on the basis of traditional droop control. To address the influence of line resistance, current-sharing layer control is introduced in Section 4.2, where the difference discrete consistency algorithm is illustrated in detail. The DDCA implements real-time tracking DC/DC converter output voltage and iteratively converges to rapidly average the values. The average value is utilized as the virtual bus voltage, so as to provide a unified reference input for each DC/DC converter, thus achieving current-sharing control. In Section 4.3, virtual bus voltage information is used as a criterion for multi-mode smooth switching of the system. A smooth switch between different modes can be achieved with voltage information only using real-time monitoring of voltage fluctuation. With the help of the strategy mentioned above, voltage stability of the system and precise load power dispatch can be guaranteed. Flow diagrams of island DC microgrid current-sharing based on Figure 3 and voltage stability control strategy are shown in Section 4.2. 


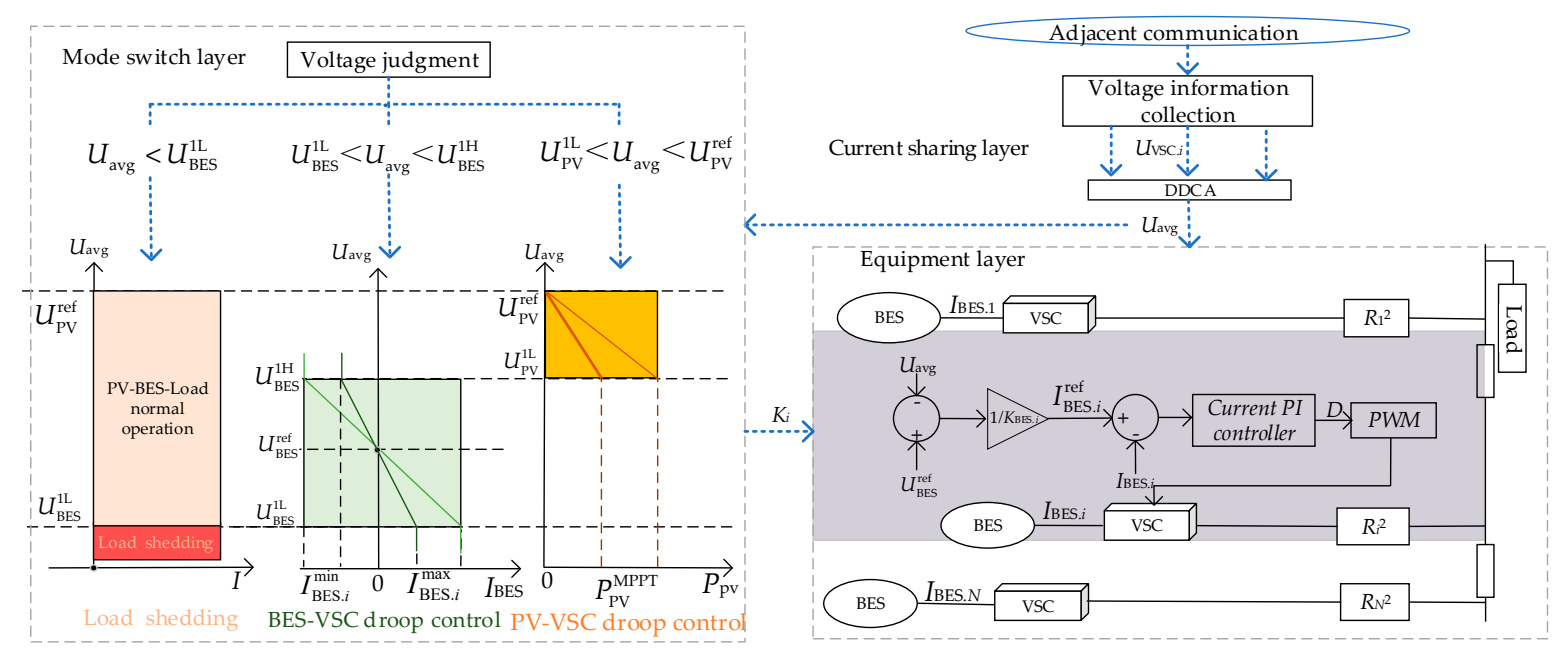

Figure 3. The hierarchical, coordinated control strategy of an island DC microgrid based on a multi-mode smooth switch.

\subsection{The First Layer: The Equipment Layer}

In the island DC microgrid system, the voltage regulation units are connected to the DC microgrid bus through a DC/DC converter, i.e., a DC microgrid voltage stability controller (VSC). To accelerate the speed of the dynamic response of droop control [22], the current-voltage (I-U) droop control structure, as indicated in Figure 4, is adopted in the system.

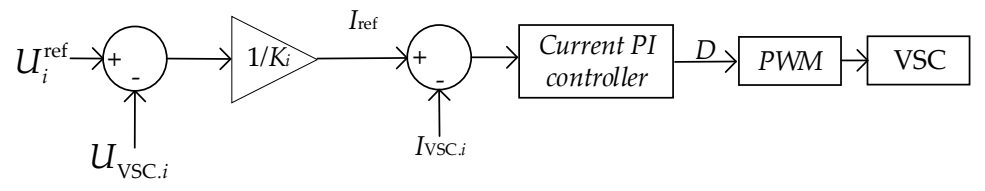

Figure 4. $I-U$ droop control diagram.

In Figure 4 , the transfer function of the PI controller is $k_{\mathrm{pi}}+k_{\mathrm{ii}} / s$. Let $k_{\mathrm{pi}}$ and $k_{\mathrm{ii}}$ represent the proportion and integral terms of current PI controller, respectively. $U_{i}^{\text {ref }}$ is the no-load voltage of VSC, i.e., the reference voltage of droop control. $U_{\mathrm{VSC} . i}$ and $I_{\mathrm{VSC} \cdot i}$ represent the output voltage and the current of the DC/DC converter, respectively. $K_{i}$ refers to the droop coefficient of VSC. $D$ represents the pulse width modulation (PWM) duty ratio.

The PV-BES load DC microgrid model utilized in this section, which takes line resistance into consideration, is indicated in Figure 5. $U_{\mathrm{PCC}}$ is the voltage of the common connection point and $R_{i}$ refers to the total resistance of the integrated line, $i$, the value of which is equal to the sum of the equivalent virtual resistance, $R_{i}^{1}$, of the droop coefficient and the integrated line resistance, $R_{i}^{2}$.

Without taking the influence of the bus piecewise resistance into consideration, the simplified structure of the island DC microgrid with a double VSC parallel operation is shown in Figure 6. In terms of conventional droop control, the output current $I_{\mathrm{VSC} \cdot i}$ of VSC, $i$, is calculated as follows:

$$
I_{\mathrm{VSC} . i}=\frac{U_{i}^{\mathrm{ref}}-U_{\mathrm{pcc}}}{R_{i}^{1}+R_{i}^{2}} \quad i \in[1,2] .
$$




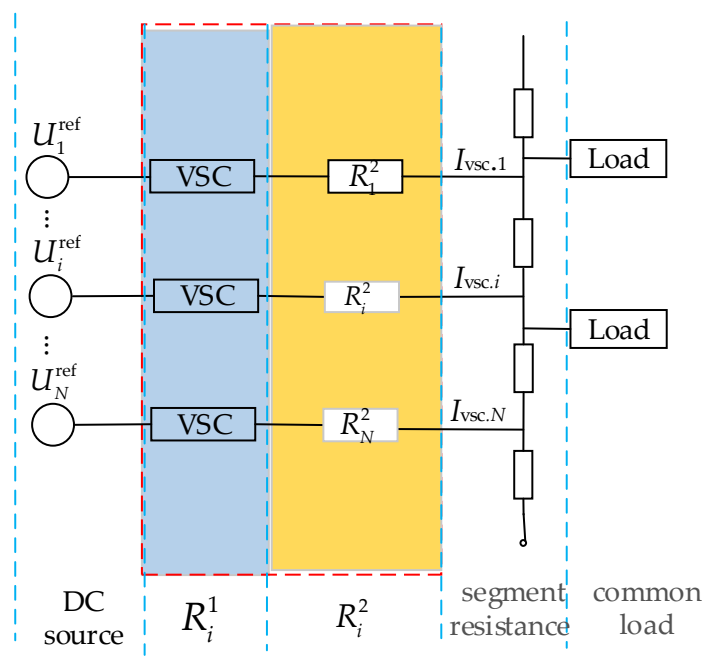

Figure 5. Parallel structure of the DC microgrid multi-converter.

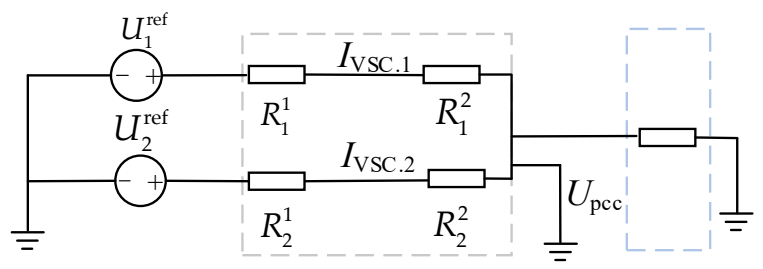

Figure 6. Simplified structure of the double parallel converters.

According to Equation (4), load power dispatch is affected by line resistance and virtual resistance. In the case of the line resistance being equal to 0 and the droop curve reference voltages of VSC being identical, the output current and droop coefficient of VSC satisfy the following proportions to achieve accurate power sharing:

$$
R_{1}^{1} I_{\mathrm{VSC} .1}=\cdots R_{i}^{1} I_{\mathrm{VSC} .} \cdot \cdots=R_{\mathrm{N}}^{1} I_{\mathrm{VSC} . \mathrm{N}}=K, i \in[1, \mathrm{~N}] .
$$

Due to the influence of line resistance, the converter $R_{i}{ }^{1}+R_{i}{ }^{2}$ is not equal in Equation (4). Considering that both the virtual resistance and line resistance under a closed-loop state are relatively low, the obvious deviation of the two resistances may lead to a considerable power imbalance and an inaccurate load power dispatch. Considering that the units in the system are connected to the DC microgrid bus in the distributed way, the bus piecewise resistance may aggravate the unbalanced dispatch of power in the system.

\subsection{The Second Layer: The Current-Sharing Layer}

According to the analysis in Section 4.1, the output voltages, $U_{\mathrm{VSC} . i}$, of VSC are different from each other because of the existence of line resistance, thus, there is no unified voltage input for VSC droop control, which results in a failure to achieve precise power dispatch. To address the influence of line resistance on the current-sharing system, researchers using traditional practices tried to increase droop coefficient to improve current-sharing [23]. This method leads to a relatively larger voltage deviation and it may still be impossible to achieve precise power dispatch. Therefore, a current-sharing layer control based on the difference discrete consistency algorithm is adopted in this paper, as indicated in Figure 3. $K_{\mathrm{BES} . i}$ refers to the droop control coefficient of $i$-th VSC, $U_{\mathrm{BES}}^{\text {ref }}$ indicates the no-load voltage of droop control of VSC, $I_{\mathrm{BES} . i}$ represents the output current of BES $i, I_{\mathrm{BES} . i}^{\max }$ refers to the maximum safety output current of BES, and $U_{\mathrm{avg}}$ indicates the average value of the terminal voltage $U_{\mathrm{VSC} . i}$ of each VSC, which are iteratively converged through DCCA. 
To reduce the requirement for the communication system, the VSC in the system only allows adjacent communication units to interact. The output voltage, $U_{\mathrm{VSC} . i}$, of each VSC is tracked in real time through DDCA and the information is rapidly converged into an average value, $U_{\text {avg. }}$. The average voltage, $U_{\mathrm{avg}}$, reflects real-time fluctuation of the DC bus voltage and provides a unified reference input for the droop control of each VSC. Thus, it is regarded as a virtual bus voltage in this section and provides a unified reference input for droop control of each VSC to achieve a balanced dispatch of load power in the system. The current-sharing layer control strategy based on DDCA is indicated in Figure 7.

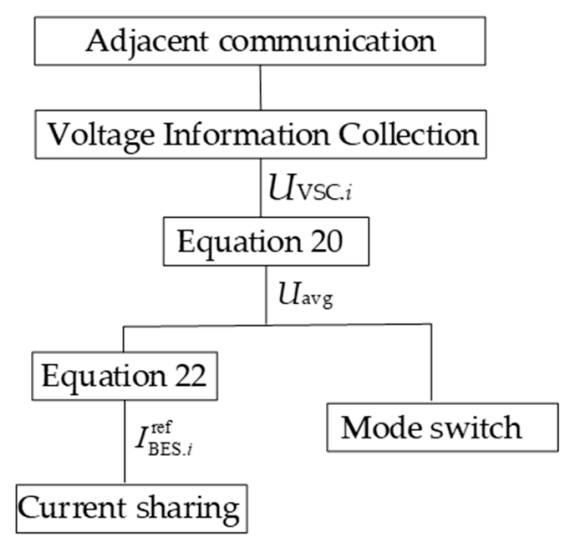

Figure 7. The current-sharing layer strategy based on the difference discrete consensus algorithm.

By utilizing the output voltage, $U_{\mathrm{VSC} . i}$, of each VSC in the island DC microgrid system as an information node and only allowing communication with adjacent VSC nodes, we find the average value, $U_{\mathrm{avg}}$, of $U_{\mathrm{VSC} . i}$ through DDCA iteration. The discrete consistency algorithm is expressed as [24]:

$$
x_{i}(k+1)=x_{i}(k)+\sum_{j \in N_{i}} a_{i j}\left(x_{j}(k)-x_{i}(k)\right) \quad i=1,2, \cdots, N,
$$

where $N$ is the total number of agent nodes, $k$ refers to the Kth iteration, $x_{i}(k)$ refers to the output of unit $i$ after the $k$ th iteration, $x_{j}(k)$ indicates the output of unit $j$ after the $k$ th iteration, $a_{i j}$ is the edge weight between node $i$ and node $j, a_{i j}=0$ when the nodes $i$ and $j$ are not neighboring nodes, and $N_{i}$ refers to the set of indexes of the agents that are connected with agent $i$.

If we consider each VSC as an agent node, then $x_{i}(k)$ in Equation (6) is represented as $U_{\mathrm{VSC} . i}(k)$ and $x_{j}(k)$ is represented as $U_{\mathrm{vsc} . j}(k)$.

After consolidation, Equation (6) can be expressed as:

$$
x_{i}(k+1)=x_{i}(k)\left(1-\sum_{j \in N_{i}} a_{i j}\right)+\sum_{j \in N_{i}} a_{i j} x_{j}(k) .
$$

It can be further indicated as:

$$
x_{i}(k+1)=W_{i i}(k) x_{i}(k)+W_{i j}(k) x_{j}(k),
$$

where $W_{i i}(k)$ and $W_{i j}(k)$ refer to the weight factor of the $k$ th iteration, respectively.

The iterative algorithm of Equation (8) is adapted as:

$$
\boldsymbol{X}(k+1)=\boldsymbol{W} \cdot \boldsymbol{X}(k) .
$$

After the $k$ th iteration:

$$
\boldsymbol{X}(k+1)=\boldsymbol{W}^{k+1} \cdot \boldsymbol{X}(0),
$$


where $\boldsymbol{X}(k)=\left[\mathrm{x}_{1}(k), x_{2}(k), \cdots x_{N}(k)\right]^{T}$ and $\boldsymbol{X}(0)=\left[x_{1}(0), x_{2}(0), \cdots x_{N}(0)\right]^{T} . \boldsymbol{W}$ refers to weight matrix of the communication network:

$$
W=\left[\begin{array}{ccc}
1-\sum_{j \in N_{1}} a_{1 j} & \cdots & a_{1 N} \\
\vdots & \ddots & \vdots \\
a_{1 N} & \cdots & 1-\sum_{j \in N} a_{N j}
\end{array}\right] .
$$

If the constant edge weight, $a$, is always adopted [25], then Equation (11) can be further indicated as:

$$
W=E-a L,
$$

where $\boldsymbol{E}$ indicates the $N$-order unit matrix, $L$ indicates the Laplacian matrix, $a$ refers to the constant edge weight, and $L$ refers to the undirected connected graph composed of the output voltage $N$ information nodes of VSCs.

$$
L_{i j}=\left\{\begin{array}{cc}
\left|N_{i}\right| & i=j \\
-1 & j \in N_{i} \\
0 & \text { other }
\end{array}\right\},
$$

where $\left|N_{i}\right|$ indicates the number of nodes involved in communication coupling with node $i$.

To guarantee that all information nodes in Equation (10) achieve iterative convergence, the following requirements must be met:

$$
\lim _{k \rightarrow \infty} \boldsymbol{W}^{k}=\frac{1}{N} \mathbf{1}^{\mathbf{T}^{\mathrm{T}}}
$$

where 1 refers to all column vectors with element of 1 .

To guarantee the convergence after iteration, Equation (14) holds only if [26]:

$$
\begin{gathered}
\mathbf{1}^{\mathrm{T}} \boldsymbol{W}=\mathbf{1}^{\mathrm{T}}, \\
\boldsymbol{W} \mathbf{1}=\mathbf{1}, \\
\rho\left(\boldsymbol{W}-\frac{1}{N} \mathbf{1} \cdot \mathbf{1}^{\mathrm{T}}\right)<1,
\end{gathered}
$$

where $\rho$ refers to the spectral radius of matrix.

The fast convergence is achieved by adjusting the value of $a$, which is valued as [25]:

$$
a=\frac{2}{\lambda_{1}(L)+\lambda_{n-1}(L)},
$$

where $\lambda_{1}(L)$ refers to the maximum eigenvalue of the Laplacian matrix, $L$, and $\lambda_{n-1}(L)$ indicates the second minimum eigenvalue of $L$.

The system stability of low-bandwidth communication (LBC) under different delays is analyzed and the results indicate that the distributed control based on LBC maintains stability, even when a relatively larger communication delay is adopted [27]. Considering that the island microgrid is relatively small, the influence of communication delay is not taken into consideration. In order to accelerate convergence and reduce fluctuation, which is caused by changes in communication topological structure and node state values, smooth convergence of the consensus algorithm is utilized. Based on the discrete consensus algorithm (DCA), the difference values of two contiguous iterations are introduced to predict future state changes. In this way, the update value for each time indicates a mixture of the predicted value and the node adjacent side calculated value. This process accelerates to skip the intermediate state of the iteration and speed up the convergence rate further. Regarding the $\beta$ 
times of the difference value, namely $\beta(X(k)-X(k-1))$, which is the predicted value in Equation (19), the DCA is updated as:

$$
\boldsymbol{X}(k+1)=\boldsymbol{W X}(k)+\beta(\boldsymbol{X}(k)-\boldsymbol{X}(k-1)) .
$$

According to Equation (19), the output voltage of each VSC satisfies:

$$
\boldsymbol{U}_{\mathrm{VSC}}(k+1)=\boldsymbol{W U}_{\mathrm{VSC}}(k)+\beta\left(\boldsymbol{U}_{\mathrm{VSC}}(k)-\boldsymbol{U}_{\mathrm{VSC}}(k-1)\right),
$$

where $\boldsymbol{U}_{\mathrm{VSC}}(k)=\left[U_{\mathrm{VSC} .1}(k), U_{\mathrm{VSC} .2}(k), \cdots U_{\mathrm{VSC} . \mathrm{N}}(k)\right]^{T}$.

According to Equation (20), the output voltage of each VSC after convergence satisfies:

$$
U_{\mathrm{avg}}=U_{\mathrm{VSC} .1}=U_{\mathrm{VSC} .2}=\cdot \cdot U_{\mathrm{VSC} . i} \cdots=U_{\mathrm{VSC} . \mathrm{N}}=\frac{1}{N} \sum_{i}^{N} U_{\mathrm{VSC} . i} .
$$

By DDCA, the droop control curve indicated in Equation (22) is developed from the conventional droop control.

$$
U_{\mathrm{avg}}=U_{\mathrm{BES}}^{\mathrm{ref}}-K_{\mathrm{BES} . i} I_{\mathrm{avg} . i},
$$

where, taking into account the maximum capacity of the BES, $I_{\text {avg. } i}$ is the BES $i$, output current under current-sharing control, namely the reference current, $\mathrm{IES} . i_{\mathrm{B}}^{\mathrm{ref}}$.

Under the identical reference voltage, $U_{\mathrm{BES}}^{\mathrm{ref}}$, and the input voltage, $U_{\mathrm{avg}}$, in the case of the output current, $I_{\mathrm{BES} . i^{\prime}}$ of BES being inversely proportional to the droop coefficient, $K_{\mathrm{BES} . i}$, current-sharing control is achieved.

$$
K_{\mathrm{BES} .1} I_{\mathrm{avg} .1}=\cdots=K_{\mathrm{BES} . i} I_{\mathrm{avg} . i}=\cdots=K_{\mathrm{BES} . \mathrm{N}} I_{\mathrm{avg} . N}
$$

After DDCA, the island DC microgrid model, which considers the line resistance in Figure 5, is improved, as shown in Figure 8.

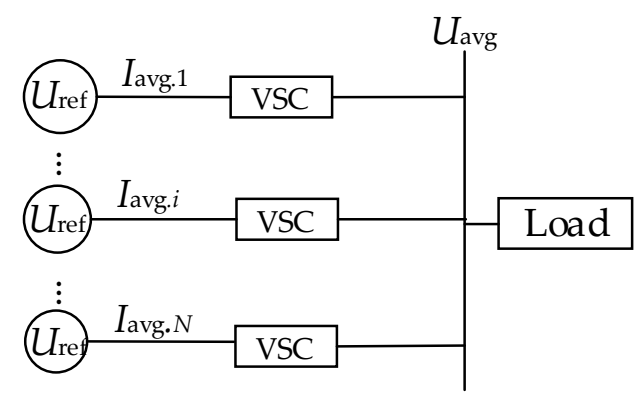

Figure 8. Equivalent model of the DC microgrid current-sharing control.

When DDCA current-sharing control is achieved, the average current, $I_{\mathrm{avg} . i}$, of BES, $I$, and the maximum output current, $I_{\mathrm{BES} . i^{\prime}}$, shall satisfies the following equation:

$$
I_{\mathrm{avg} . i}^{\mathrm{un}}=\frac{I_{\mathrm{avg} . i}}{I_{\mathrm{BES} . i}^{\max }}
$$

where $I_{\mathrm{avg} . i}^{\mathrm{un}}$ is the average unit current under current sharing control, which is used to indicate the load rate of BES.

$$
I_{\mathrm{avg} .1}^{\mathrm{un}}=I_{\mathrm{avg} .2}^{\mathrm{un}}=\cdots I_{\mathrm{avg} . i}^{\mathrm{un}} \cdots=I_{\mathrm{avg} . N}^{\mathrm{un}}=I_{\mathrm{avg}}^{\mathrm{un}}
$$

To verify the effectiveness of the current-sharing strategy, a topological structure with five information nodes, as indicated in Figure 9a, is established in the paper. Figure $9 \mathrm{~b}$ shows the corresponding Laplacian matrix, $L$, of this structure. 


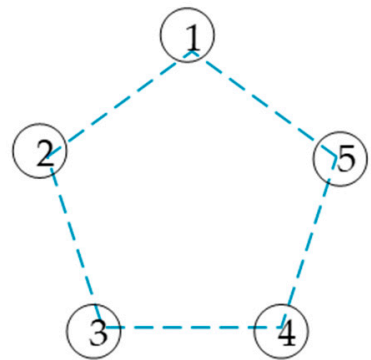

(a)

$$
\boldsymbol{L}=\left[\begin{array}{ccccc}
2 & -1 & 0 & 0 & -1 \\
-1 & 2 & -1 & 0 & 0 \\
0 & -1 & 2 & -1 & 0 \\
0 & 0 & -1 & 2 & -1 \\
-1 & 0 & 0 & -1 & 2
\end{array}\right]
$$

(b)

Figure 9. Communication topology and its Laplacian matrix. (a) A topological structure with five information nodes; (b) Laplacian matrix.

The system starts with $\mathbf{X}(0)=[1,2,3,4,5]^{T}$. The eigenvalues of $\boldsymbol{L}$ are $[0,1.382,1.382,3.618,3.618]^{T}$. The convergence when $a=2 / 5, a=1 / 5$, and $a=1 / 10$ is verified in this paper by Matlab simulation.

Figure 10 shows that $a=2 / 5$ has the fastest iteration speed and the lowest fluctuation.

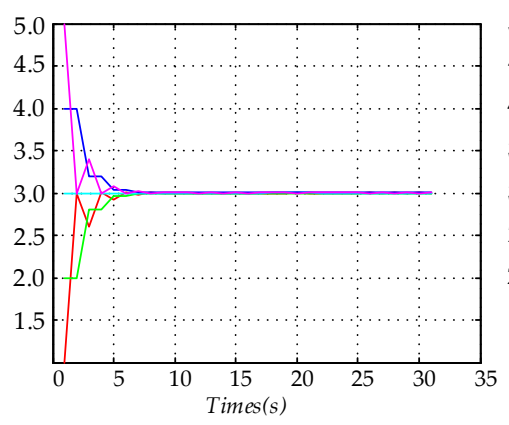

(a)

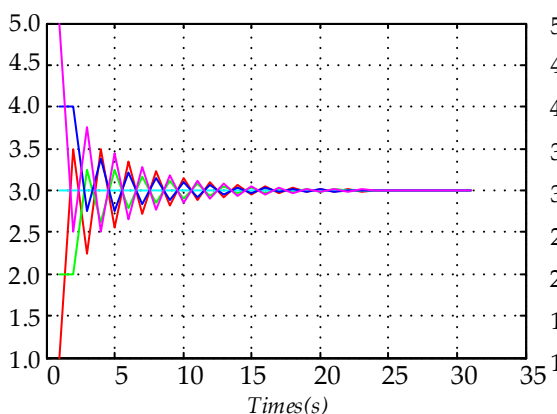

(b)

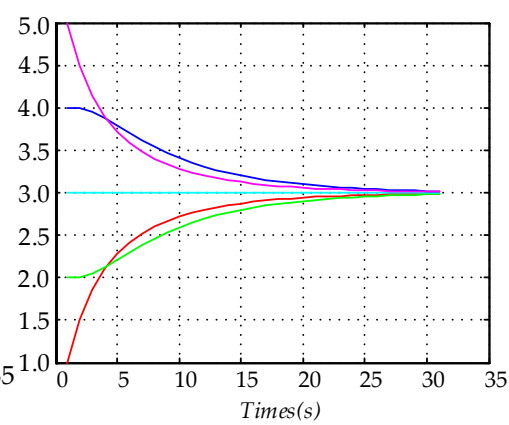

(c)

Figure 10. Converging speed comparison under different constant weights, $a$. (a) Consensus dynamic at $a=2 / 5$; (b) Consensus dynamic at $a=1 / 2$; (c) Consensus dynamic at $a=1 / 10$.

\subsection{The Third Layer: The Multi-Mode Smooth Switch Layer}

Considering that the power consumption of line resistance, $P_{\mathrm{L}}$, is hard to acquire accurately, it is not appropriate to utilize the real-time power of the system units in Section 3 as the criterion for the multi-mode smooth switch layer, which may lead to serious deviation. When using DC bus voltage as the key index for the system operation, the power status of the DC microgrid system is reflected in real time. The DC bus voltage is of great significance for the multi-mode smooth switch. Meanwhile, the average voltage, $U_{\text {avg }}$, introduced in Section 4.2 , reflects the true level of bus voltage. Thus, average voltage, $U_{\mathrm{avg}}$, is regarded as a virtual bus voltage in this section to provide a unified voltage criterion for mode switching of the system units. At the same time, it provides a unified reference input for all VSCs. A multi-mode smooth switch control strategy, based on virtual bus voltage information, is proposed in this paper, as indicated in Figure 3. Among them, $U_{\mathrm{BES}}^{\mathrm{ref}}$ refers to the no-load voltage of BES-VSC, $U_{\mathrm{BES}}^{1 \mathrm{~L}}$ and $U_{\mathrm{BES}}^{1 \mathrm{H}}$ indicate the scope of the droop voltage regulation of BES-VSC, $U_{\mathrm{PV}}^{\text {ref }}$ represents the no-load voltage of droop control of PV-VSC, $U_{\mathrm{PV}}^{1 \mathrm{~L}}$ indicates the minimum voltage of droop control of PV-VSC, and $P_{\mathrm{PV}}$ refers to the output power of PV.

The multi-mode smooth switch strategy based on the virtual bus voltage information is shown in Figure 11. 


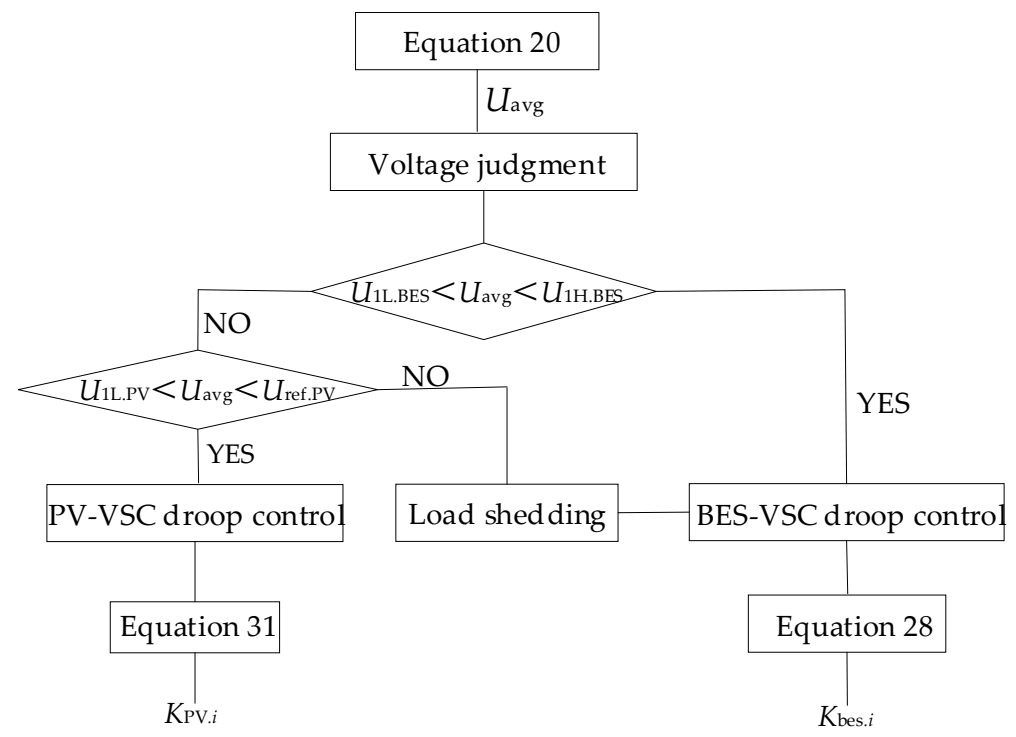

Figure 11. Multi-mode switch strategy based on virtual bus voltage information.

(1) Mode 1: BES-VSC droop control

According to Figure 11, to fully utilize photovoltaic energy, MPPT control is adopted under this mode and the bus voltage is regulated through droop control of BES-VSC. As indicated in the figure, the admissible fluctuation scope of bus voltage is between $U_{\mathrm{BES}}^{1 \mathrm{~L}}$ and $U_{\mathrm{BES}}^{1 \mathrm{H}}$. The virtual bus voltage, $U_{\text {avg, }}$ is introduced as the criterion for the multi-mode smooth switch and the reference input for each VSC. Thus, the $I-U$ droop control adopted under this mode is expressed as:

$$
I_{\mathrm{avg} . i}=\frac{U_{\mathrm{BES}}^{\mathrm{ref}}-U_{\mathrm{avg}}}{k_{\mathrm{BES} . i}}=I_{\mathrm{BES} . i}^{\mathrm{ref}} .
$$

To fully utilize the voltage regulation capacity of BES, in case of the virtual bus voltage $U_{\text {avg }}$ exceeding the fluctuation scope, all VSCs implement multi-mode smooth switching simultaneously. This means that, when $U_{\text {avg }}$ reaches the bounds of the droop voltage regulation area of BES-VSC, the output current of each BES, $I_{\mathrm{avg} . i^{\prime}}^{\mathrm{un}}$ meets the following equations:

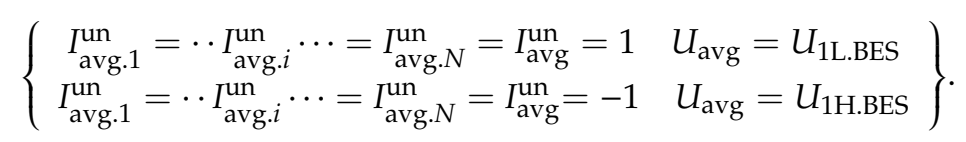

To achieve multi-mode smooth switching, the BES-VSC droop coefficient, $K_{\mathrm{BES} . i}$, is set as:

$$
K_{\mathrm{BES} . i}=\frac{U_{\mathrm{BES}}^{\mathrm{ref}}-U_{\mathrm{BES}}^{1 \mathrm{~L}}}{I_{\mathrm{BES} . i}^{\max }}=\frac{U_{\mathrm{BES}}^{\mathrm{ref}}-U_{\mathrm{BES}}^{1 \mathrm{H}}}{I_{\mathrm{BES} . i}^{\min }} .
$$

(2) Mode 2: PV-VSC droop control

According to Figure 11, when there is a surplus of power in the system, the virtual bus voltage exceeds the maximum value of energy storage in the droop voltage regulation area and the system voltage enters into the PV-VSC droop voltage regulation area. Under this mode, BES adopts a constant output current, $I_{\mathrm{BES} .{ }^{\mathrm{m}} \text {, }}$ and the bus voltage is under the droop control regulation of PV-VSC. The fluctuation scope for the virtual bus voltage under the droop control voltage regulation of PV-VSC is between $U_{\mathrm{PV}}^{1 \mathrm{~L}}$ and $U_{\mathrm{PV}}^{\mathrm{ref}}$. As Figure 3 shows, to achieve a smooth switch between mode 1 and mode 2, when $P_{\mathrm{PV} . i}$ reaches $P_{\mathrm{PV} . i}^{\mathrm{MPPT}}$, namely:

$$
P_{\mathrm{PV} . i} / P_{\mathrm{PV} . i}^{\mathrm{MPPT}}=1,
$$


the virtual bus voltage, $U_{\mathrm{avg}}$, satisfies the following equation:

$$
U_{\mathrm{avg}}=U_{\mathrm{BES}}^{1 \mathrm{H}}=U_{\mathrm{PV}}^{1 \mathrm{~L}} \text {. }
$$

Similarly, to achieve multi-mode smooth switching between mode 1 and mode 2, the droop control coefficient, $K_{\mathrm{PV} . i}$, satisfies the following equation:

$$
K_{\mathrm{PV} . i}=\frac{U_{\mathrm{PV}}^{\mathrm{ref}}-U_{\mathrm{PV}}^{1 \mathrm{~L}}}{P_{\mathrm{PV} . i}^{\mathrm{MPPT}}}=\frac{U_{\mathrm{PV}}^{\mathrm{ref}}-U_{\mathrm{BES}}^{1 \mathrm{H}}}{P_{\mathrm{PV} . i}^{\mathrm{MPT}}} .
$$

(3) Mode 3: Load shedding

According to Figure 11, in the case of the system having power vacancy and this leading to a reduction in bus voltage, the multi-mode switch strategy is initiated when the virtual bus voltage is lower than $U_{\mathrm{BES}}^{1 \mathrm{~L}}$. A load with a lower priority level is shed to complete load-shedding control and guarantee stable operation of the system. When load-shedding control is implemented, the total load power of the system is reduced and the system operation returns to the droop control voltage regulation area of BES-VSC, as indicated in Figure 3.

\section{Example Simulation Analysis}

To verify the effectiveness of the hierarchical, coordinated control strategy of the island DC microgrid under multiple modes proposed in this paper, the island DC microgrid indicated in Figure 5 is established in PSCAD/EMTDC. This DC microgrid contains four groups of battery energy storage units, from BES1 to BES 4, including one group of common DC load and one group of photovoltaic power generation array, and achieves integrated operation through a DC/DC converter. The communication topological structure among the four groups of the energy storage system is indicated in Figure 12. Its communication matrix eigenvalue $\lambda$ is [0,2,2,4]. The value of $a$ is obtained from Equation (18) as 1/3. The energy storage maximum current output ratio is 1:2:3:4. In addition, the bus piecewise resistances are $0.5,0.2$, and $0.4 \Omega$, respectively, and the energy storage no-load voltage, $U_{\mathrm{BES},}^{\mathrm{ref}}$, is $380 \mathrm{~V}$. Furthermore, the voltage range of the multi-mode switch from $U_{\mathrm{BES}}^{1 \mathrm{~L}}$ and $U_{\mathrm{BES}}^{1 \mathrm{H}}$ is between 370 and $385 \mathrm{~V}$ and for $U_{\mathrm{PV}}^{1 \mathrm{~L}}$ and $U_{\mathrm{PV}}^{\text {ref }}$. the range is between 385 and $390 \mathrm{~V}$. The operational situation of this DC microgrid is indicated in Table 1. Simulation analysis is carried out for this DC microgrid in terms of the source load fluctuation. To verify the effectiveness of the control strategy proposed in this paper under different situations, the simulations of all six situations listed in Table 1 are achieved within $0-8 \mathrm{~s}$ sequentially.

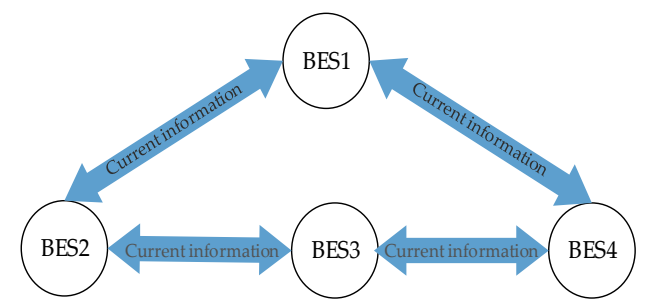

Figure 12. Topological structure of four groups of energy storage communication.

Table 1. Operational situations of DC microgrid.

\begin{tabular}{ccc}
\hline Situation & Operation Mode & Operation Time/s \\
\hline 1 & 1 & $0-2.5 \mathrm{~s}$ \\
2 & $1-3-1$ & $2.5-4.4 \mathrm{~s}$ \\
3 & $1-2$ & $4.4-6.2 \mathrm{~s}$ \\
4 & $2-1$ & $6.2-6.8 \mathrm{~s}$ \\
5 & 1 & $6.8-8 \mathrm{~s}$ \\
6 & 1 & $0-2.5 \mathrm{~s}$ \\
\hline
\end{tabular}




\subsection{Situation 1}

When the DC microgrid operates normally under Situation 1, the photovoltaic array uses MPPT control and the four groups of energy storage units adopt droop control to regulate the voltage. Before $1.8 \mathrm{~s}$, both the photovoltaic array output and common load are 0 , therefore the system is under a no-load state. At $1.8 \mathrm{~s}$, the common DC load increases to $8 \mathrm{~kW}$, with the four groups of energy storage units adopting droop control voltage regulation, consequently reducing system voltage. After $2 \mathrm{~s}$, the DDCA is initiated to complete current-sharing control.

According to Figure 13, current-sharing control does not occur in the first $2 \mathrm{~s}$. The unit currents of $I_{\mathrm{BES} .1}^{\mathrm{un}}$ and $I_{\mathrm{BES} .2}^{\mathrm{un}}$ are over 1.0, which means these two energy storage units are overloaded at this point. Meanwhile, deviation exists between the bus voltages at different nodes, which makes it impossible to implement accurate multi-mode switching. After $2 \mathrm{~s}$, the system initiates the current-sharing control strategy and the $I_{\mathrm{avg}}^{\mathrm{un}}$ of the four groups of energy storage units maintain the current at 0.7. In addition, the output current of the energy storage units is inversely proportional to the droop coefficient, allowing the system to achieve current-sharing. The virtual bus voltage gradually converges and is maintained at $373 \mathrm{~V}$, which is within the range of the energy storage droop voltage regulation of mode 1.

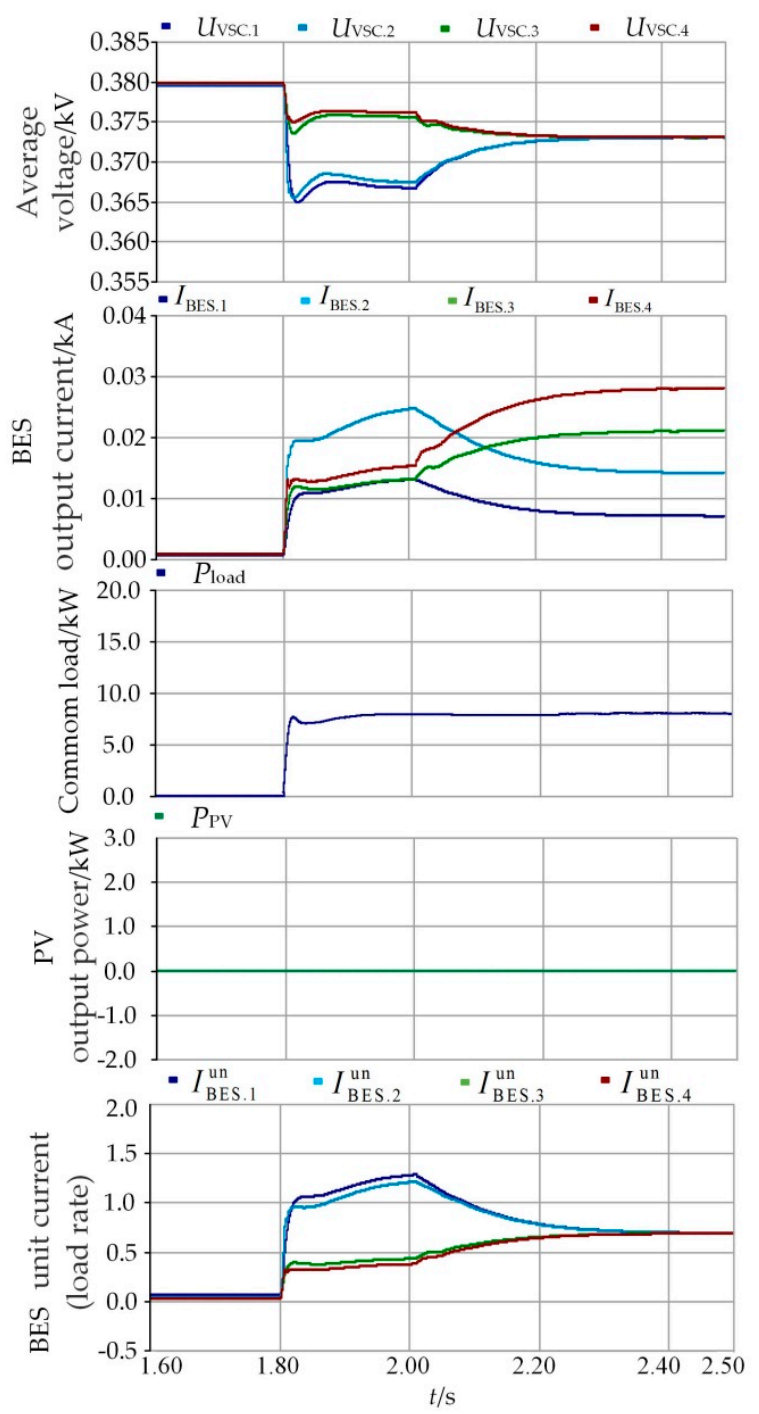

Figure 13. Verification for the effectiveness of DDCA convergence. 


\subsection{Situation 2}

At $2.5 \mathrm{~s}$, the photovoltaic array output increases to $6 \mathrm{~kW}$ and the voltage also rises. At $3 \mathrm{~s}$, the DC load increases by $8 \mathrm{~kW}$ while the voltage reduces to $U_{\mathrm{BES}}^{1 \mathrm{~L}}$. At $3.6 \mathrm{~s}$, the DC load increases by $2 \mathrm{~kW}$; at this point, the system has a power vacancy, with the voltage level lower than $U_{\mathrm{BES}}^{1 \mathrm{~L}}$. At $3.65 \mathrm{~s}$, the multi-mode smooth switch strategy is initiated and the system enters into mode 3, where load-shedding voltage regulations exist, removing $8 \mathrm{~kW}$ load sequentially, according to priority level. After this, the bus voltage rises again to the range of mode 1 energy storage droop control.

As shown in Figure 14, at $2.5 \mathrm{~s}$, the $I_{\mathrm{avg}}^{\mathrm{un}}$ of the four groups of energy storage units drops to 0.17 , while the virtual bus voltage rises to $378.3 \mathrm{~V}$ and the system completes current-sharing and voltage stability control. At $3 \mathrm{~s}$, the DC load increases and the $I_{\mathrm{avg}}^{\mathrm{un}}$ of energy storage unit increases to 1; the voltage at this point stabilizes at $U_{\mathrm{BES}}^{1 \mathrm{~L}}$. At $3.6 \mathrm{~s}$, the load increase leads to a system power vacancy and the bus voltage drops continuously to a level lower than $U_{\mathrm{BES}}^{1 \mathrm{~L}}$. At $3.65 \mathrm{~s}$, the multi-mode switch strategy is initiated and the system starts the load-shedding voltage regulation process to complete load-shedding control; at this point the $I_{\mathrm{avg}}^{\mathrm{un}}$ of the energy storage unit is maintained at 0.32 and the virtual bus voltage rises to $376.8 \mathrm{~V}$. The system enters into the energy storage unit droop control range under mode 1.

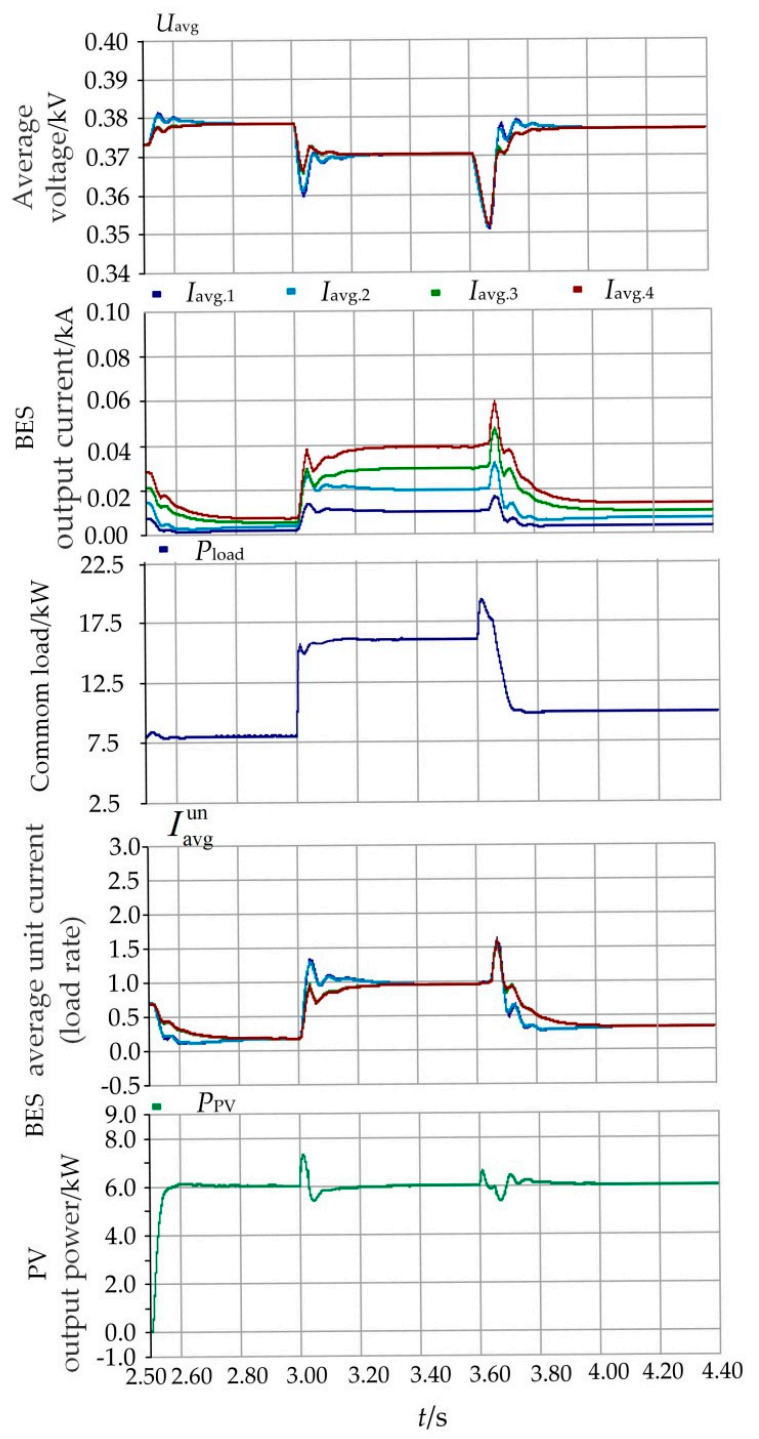

Figure 14. Simulation waveform of Situation 2. 


\subsection{Situation 3}

At $4.6 \mathrm{~s}$, the DC load falls by $10 \mathrm{~kW}$ and the virtual bus voltage rises continuously, exceeding the energy storage unit no-load voltage of $U_{\mathrm{BES}}^{\text {ref }}$; in addition, the energy storage system converts from discharge mode to charge mode. At $5.0 \mathrm{~s}$, the photovoltaic output increases by $2.5 \mathrm{~kW}$ and the virtual bus voltage rises to $U_{\mathrm{BES}}^{1 \mathrm{H}}$. At $5.5 \mathrm{~s}$, the photovoltaic output enhances by $2.5 \mathrm{~kW}$ with excess system power and the virtual bus voltage exceeds $U_{\mathrm{BES}}^{1 \mathrm{H}}$. At $5.51 \mathrm{~s}$, the multi-mode switch strategy is initiated and the photovoltaic array switches from MPPT control to droop control; the virtual bus voltage is restored to the range of photovoltaic droop voltage regulation.

As shown in Figure 15, at the time of $4.6 \mathrm{~s}$, the DC load drops and the four groups of energy storage units adopt droop control; the virtual bus voltage rises to $383.5 \mathrm{~V}$ and the $I_{\mathrm{avg}}^{\mathrm{un}}$ of the energy storage unit drops to -0.7 . At $5.0 \mathrm{~s}$, the photovoltaic output rises, with the $I_{\mathrm{avg}}^{\mathrm{un}}$ of the energy storage unit dropping to -1.0 and the bus voltage reaching $385 \mathrm{~V}$. At $5.5 \mathrm{~s}$, with the increase in photovoltaic output, the system contains excess power and the virtual bus voltage rises continuously to exceed $U_{\mathrm{BES}}^{1 \mathrm{H}}$. At $5.51 \mathrm{~s}$, the multi-mode switch strategy is initiated and the photovoltaic unit enters into a limited power mode, with the virtual bus voltage rising again to $386 \mathrm{~V}$ and the system voltage entering into the photovoltaic droop voltage regulation range.
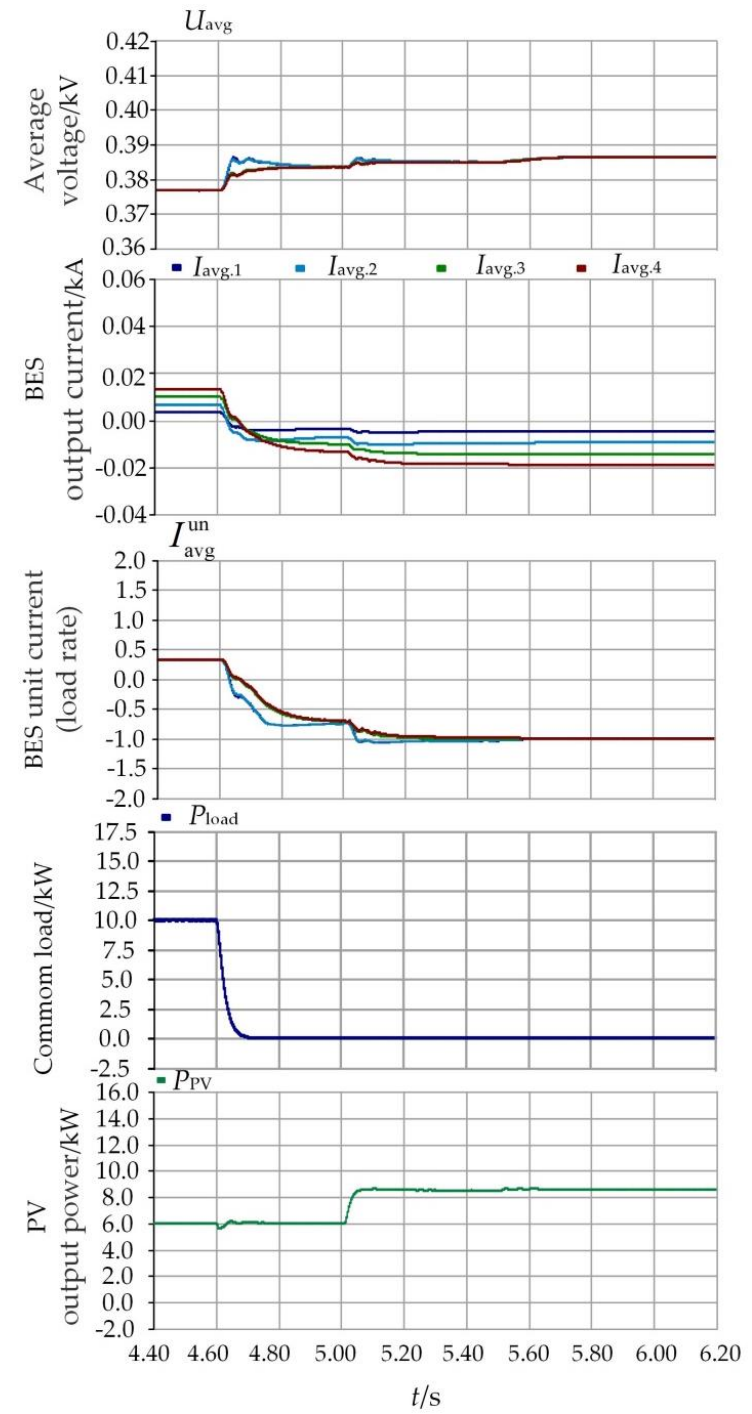

Figure 15. Simulation waveform of Situation 3. 


\subsection{Situation 4}

At $6.3 \mathrm{~s}$, with the DC load increasing by $12 \mathrm{~kW}$, the photovoltaic output rises and the virtual bus voltage is lower than $U_{\mathrm{PV}}^{1 \mathrm{~L}}$. As the multi-mode switch strategy is initiated, the system is under the energy storage droop control range of mode 1. In addition, as the photovoltaic unit initiates MPPT control, the energy storage unit adopts the droop control voltage regulation and the output current is positive.

According to Figure 16, at $6.3 \mathrm{~s}$, the DC load increases and the virtual bus voltage is lower than $U_{\mathrm{PV}}^{1 \mathrm{~L}}$. As the multi-mode strategy is initiated, the voltage locates within the droop control range of the energy storage system and is maintained at $379 \mathrm{~V}$; the $I_{\text {avg }}^{\mathrm{un}}$ of the energy storage unit is 0.1 . The photovoltaic array converts from droop control to the MPPT mode and the system operates in a stable manner under mode 1 .

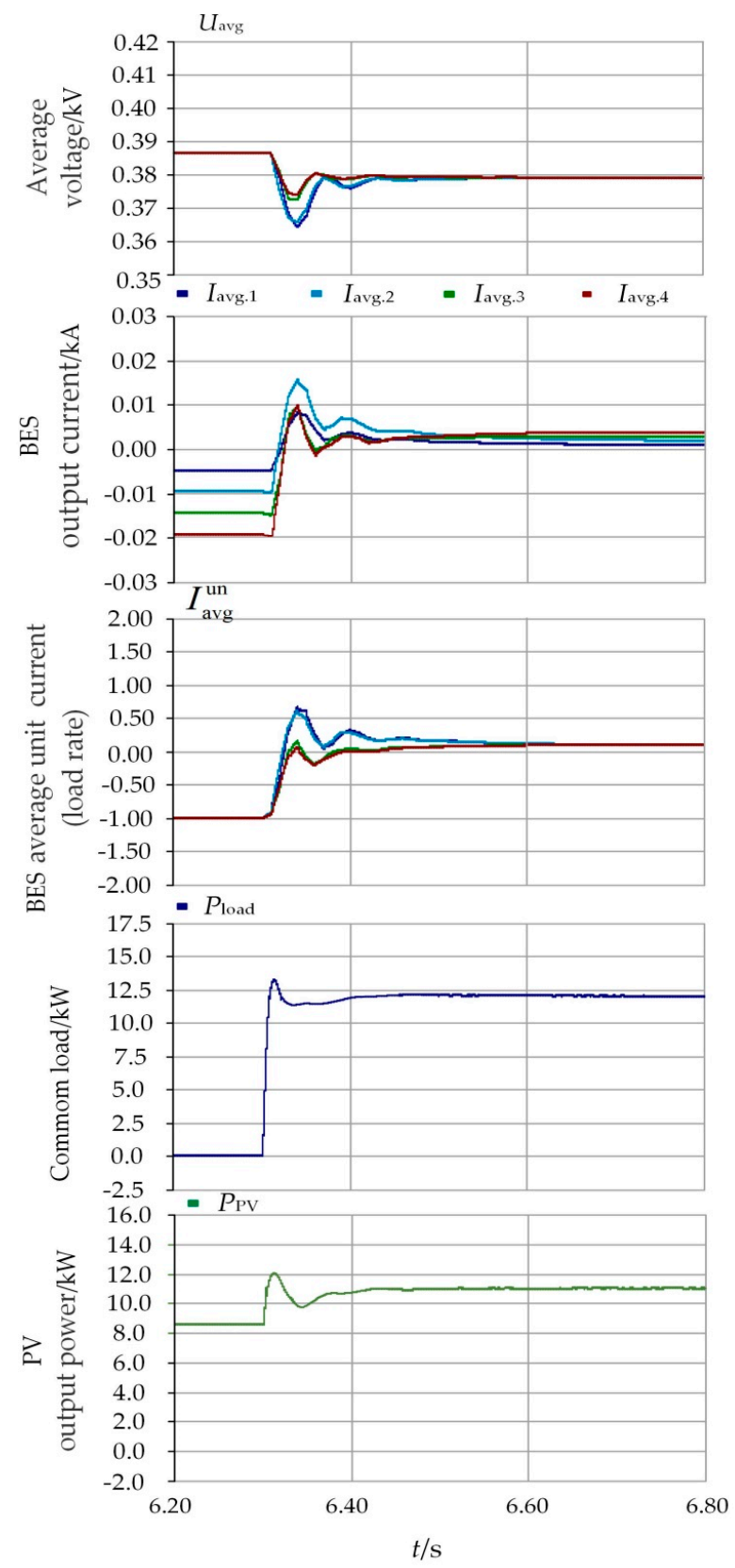

Figure 16. Simulation waveform of Situation 4. 


\subsection{Situation 5}

At $7.0 \mathrm{~s}$, the output of public DC loads and photovoltaic units remains unchanged and the four energy storage units complete discharge and exit the operation. The system voltage is controlled by the remaining energy storage unit by droop control.

According to Figure 17, at $7.0 \mathrm{~s}, I_{\mathrm{avg}}^{\mathrm{un}}$ is reduced to 0 and the system voltage is adjusted by the droop control of the remaining three BES units. The virtual bus voltage is reduced to $378.4 \mathrm{~V}$ and $I_{\text {avg }}^{\text {un }}$ is raised to 0.16 . The system voltage remains in the energy storage sagging pressure area.
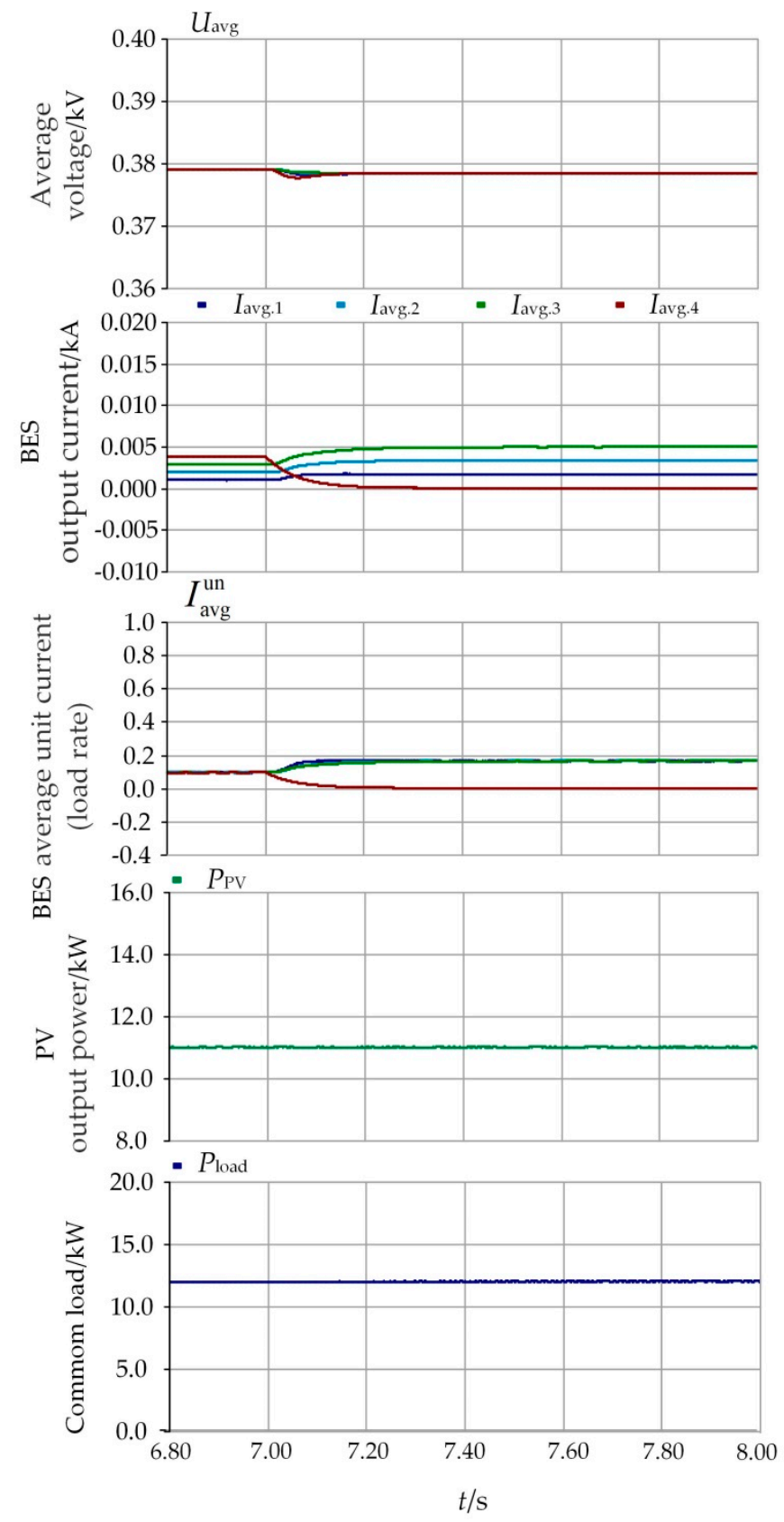

Figure 17. Simulation waveform of Situation 5.

\subsection{Situation 6}

Taking photovoltaic and load fluctuation into consideration in Situation $1-5$, to verify the robustness of current-sharing control when the disturbance of line resistance is introduced, Situation 6 investigates the current-sharing conditions under a $+20 \%$ and $-30 \%$ line resistance disturbance. 
Figure $18 \mathrm{a}, \mathrm{b}$ indicates the response curves with disturbances of $+20 \%$ and $-30 \%$, respectively.

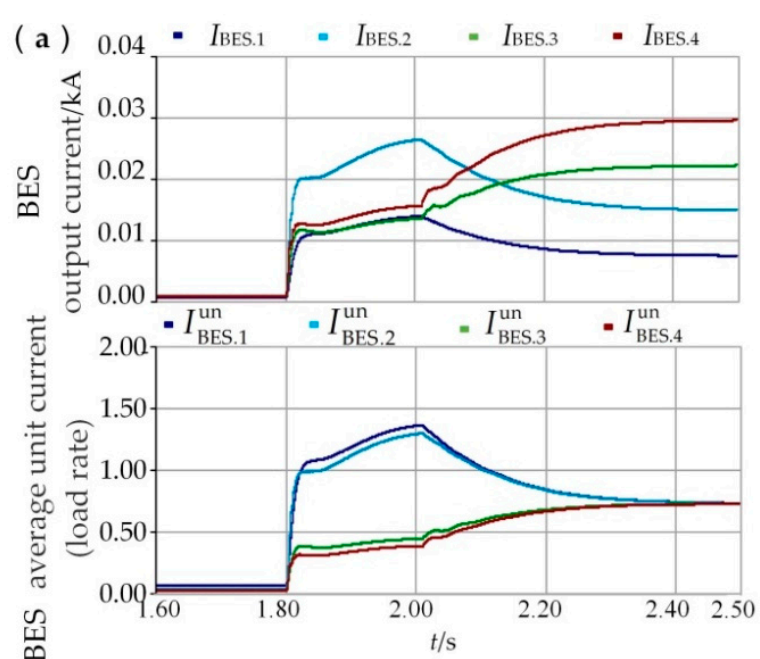

(a)

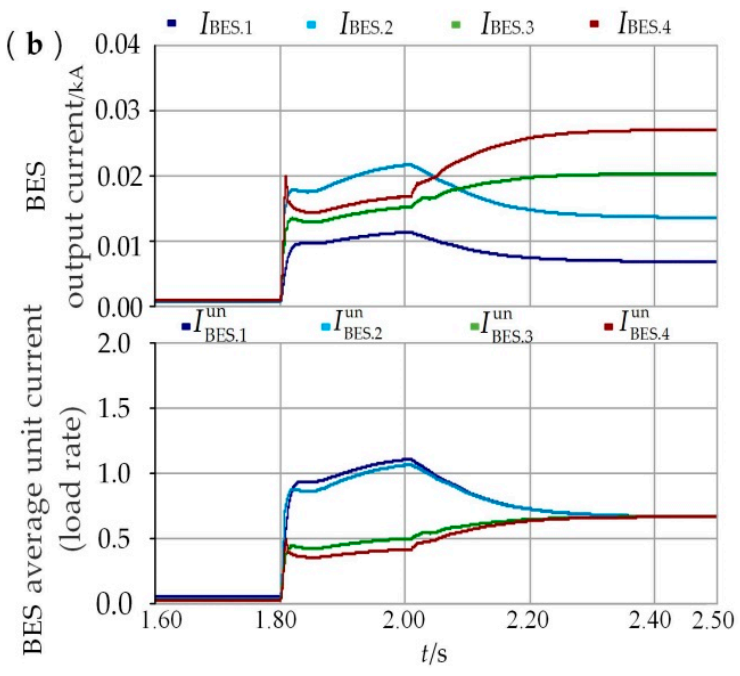

(b)

Figure 18. Simulation waveform of Situation 6. (a) $+20 \%$ disturbance; (b) $-30 \%$ disturbance.

According to Figure 18, in the case of the line resistance being disturbed, the system still achieves rapid current-sharing. This verifies the robustness of the strategy when disturbances are introduced.

\section{Conclusions}

A hierarchical, coordinated control strategy of an island DC microgrid based on a multi-mode switch is proposed in this paper. To solve the inaccurate load dispatch caused by line resistance and guarantee system voltage stability, this work proposes the establishment of a three-layer control structure, including an equipment layer, a current-sharing layer, and a mode switching layer, on the basis of conventional droop control. The simulation verification is carried out based on PSCAD/EMTDC and the results indicate that:

(1) In the mode switching layer, the control strategy solves the bus voltage deviation problem caused by line resistance and the influence of power loss on a multi-mode switch. Based on virtual bus voltage information, the smooth switch between different operation modes of an island DC microgrid is achieved.

(2) In the current-sharing layer, which avoids centralized control and upper energy management, the control strategy utilizes DDCA to track the output of each VSC unit in real time, eliminating the influence of line resistance and providing reliable virtual bus voltage information for the equipment layer and the mode switching layer.

(3) In the equipment layer, the output current of each unit with current-sharing control achieves accurate load power dispatch. In addition, the control strategies of each unit are regulated in terms of the fluctuation of virtual bus voltage information to achieve system voltage stability.

Author Contributions: Conceptualization, Z.Z., J.Z. and Y.H.; Formal analysis, Z.Z. and J.Z.; Methodology, Z.Z. and J.Z.; Software, Z.Z.; Writing—original draft, Z.Z., J.Z. and Y.H.; Supervision, J.Z. and Y.H.; Revision, Z.Z., J.Z. and Y.Z. All authors have contributed to the editing and proofreading of this paper.

Funding: This research was funded by National Natural Science Foundation of China, grant number 51867005. The Science and Technology Foundation of Guizhou Province, grant number [2016]1036. Guizhou Province Science and Technology Innovation Talent Team Project, grant number [2018]5615. Guizhou Province Reform Foundation for Postgraduate Education, grant number [2016]02, The Science and Technology Foundation of Guizhou Province, grant number [2018]5781.

Conflicts of Interest: The authors declare no conflicts of interest 


\section{References}

1. Arefifar, S.A.; Mohamed, Y.A.R.I. DG Mix, Reactive sources and energy storage units for optimizing microgrid reliability and supply security. IEEE Trans. Smart Grid 2014, 5, 1835-1844. [CrossRef]

2. Mengelkamp, E.; Gärttner, J.; Rock, K.; Kessler, S.; Orsini, L.; Weinhardt, C. Designing microgrid energy markets: A case study: The brooklyn microgrid. Appl. Energy 2018, 210, 870-880. [CrossRef]

3. Zhang, J.; Xiong, G.; Meng, K.; Yu, P.; Yao, G.; Dong, Z. An improved probabilistic load flow simulation method considering correlated stochastic variables. Int. J. Electr. Power Energy Syst. 2019, 111, $260-268$. [CrossRef]

4. Loh, P.C.; Li, D.; Chai, Y.K.; Blaabjerg, F. Autonomous operation of hybrid microgrid with AC and DC subgrids. IEEE Trans. Power Electron. 2013, 28, 2214-2223. [CrossRef]

5. Papadimitriou, C.N.; Zountouridou, E.I.; Hatziargyriou, N.D. Review of hierarchical control in DC microgrids. Electr. Power Syst. Res. 2015, 122, 159-167. [CrossRef]

6. Lotfi, H.; Khodaei, A. AC Versus DC microgrid planning. IEEE Trans. Smart Grid 2015, 8, 296-304. [CrossRef]

7. Shuai, Z.; Fang, J.; Ning, F.; Shen, Z.J. Hierarchical structure and bus voltage control of DC microgrid. Renew. Sustain. Energy Rev. 2018, 82, 3670-3682. [CrossRef]

8. Tucci, M.; Riverso, S.; Vasquez, J.C.; Guerrero, J.M.; Ferrari-Trecate, G. A decentralized scalable approach to voltage control of dc islanded microgrids. IEEE Trans. Control Syst. Technol. 2015, 24, 1965-1979. [CrossRef]

9. Guo, L.; Feng, Y.; Li, X.; Wang, C. Stability analysis of a DC microgrid with master-slave control structure. In Proceedings of the 2014 IEEE Energy Conversion Congress and Exposition (ECCE), Pittsburgh, PA, USA, 14-18 September 2014; pp. 5682-5689.

10. Guerrero, J.M.; Vasquez, J.C.; Matas, J.; De Vicuna, L.G.; Castilla, M. Hierarchical Control of Droop-Controlled AC and DC Microgrids-A General Approach toward Standardization. IEEE Trans. Ind. Electron. 2011, 58, 158-172. [CrossRef]

11. Gu, Y.; Xiang, X.; Li, W.; He, X. Mode-Adaptive Decentralized Control for Renewable DC Microgrid with Enhanced Reliability and Flexibility. IEEE Trans. Power Electron. 2014, 29, 5072-5080. [CrossRef]

12. Khorsandi, A.; Ashourloo, M.; Mokhtari, H. A Decentralized Control Method for a Low-Voltage DC Microgrid. IEEE Trans. Energy Convers. 2014, 29, 793-801. [CrossRef]

13. Zhou, T.; Francois, B. Energy management and power control of a hybrid active wind generator for distributed power generation and grid integration. IEEE Trans. Ind. Electron. 2011, 58, 95-104. [CrossRef]

14. Mi, Y.; Zhang, H.; Fu, Y.; Wang, C.; Loh, P.C.; Wang, P. Intelligent power sharing of dc isolated microgrid based on fuzzy sliding mode droop control. IEEE Trans. Smart Grid 2019, 10, 2396-2406. [CrossRef]

15. Yazdanian, M.; Mehrizi-Sani, A. Distributed Control Techniques in Microgrids. IEEE Trans. Smart Grid 2014, 5, 2901-2909. [CrossRef]

16. Eriksson, R.; Beerten, J.; Ghandhari, M.; Belmans, R. Optimizing DC Voltage Droop Settings for AC/DC System Interactions. IEEE Trans. Power Deliv. 2014, 29, 362-369. [CrossRef]

17. Beerten, J.; Belmans, R. Analysis of Power Sharing and Voltage Deviations in Droop-Controlled DC Grids. IEEE Trans. Power Syst. 2013, 28, 4588-4597. [CrossRef]

18. Mi, Y.; Wang, Y.; Zhu, Y.; Fu, Y.; Wang, C. Coordinated control for autonomous dc microgrid with dynamic load power sharing. Power Syst. Technol. 2017, 41, 440-447.

19. Wang, P.; Wang, W.; Meng, N.; Wu, Y. Unified Control Strategy of Islanding and Grid-connected Operations for DC Microgrid. Proc. CSEE 2015, 35, 4388-4396.

20. Yang, J.; Jin, X.; Wu, X.; Acuna, P.; Aguilera, R.P. Decentralised control method for DC microgrids with improved current sharing accuracy. LET Gener. Transm. Distrib. 2017, 11, 696-706. [CrossRef]

21. Ziwen, L.; Shihong, M.; Zhihua, F.; Kaiyun, C.; Yilong, K. Accurate Power Allocation and Zero Steady-State Error Voltage Control of the Islanding DC Microgird Based on Adaptive Droop Characteristics. Trans. China Electrotech. Soc. 2019, 34, 795-806.

22. Wang, H.; Han, M.; Han, R.; Guerrero, J.; Vasquez, J. A Decentralized Current-Sharing Controller Endows Fast Transient Response to Parallel DC-DC Converters. IEEE Trans. Power Electron. 2017, 33, 4362-4372. [CrossRef]

23. Zhu, S.; Wang, F.; Guo, F.; Wang, Q.; Gao, Y. Overview of Droop Control in DC Microgrid. Proc. CSEE 2018, 38, 72-84. 
24. Gao, Y.; Ai, Q.; Yousif, M.; Muhammad, W. Source-load-storage consistency collaborative optimization control of flexible DC distribution network considering multi-energy complementarity. Int. J. Electr. Power Energy Syst. 2019, 107, 273-281. [CrossRef]

25. Meng, L.; Dragicevic, T.; Guerrero, J.M.; Vasquez, J.C. Dynamic Consensus Algorithm Based Distributed Global Efficiency Optimization of a Droop Controlled DC Microgrid. In Proceedings of the 2014 IEEE International Energy Conference, Cavtat, Croatia, 13-16 May 2014; IEEE Press: Dubrovnik, Croatia, 2014; pp. 1276-1283.

26. Oreshkin, B.N.; Coates, M.J.; Rabbat, M.G. Optimization and Analysis of Distributed Averaging with Short Node Memory. IEEE Trans. Signal Process. 2010, 58, 2850-2865. [CrossRef]

27. Lu, X.; Guerrero, J.M.; Sun, K.; Vasquez, J.C. An Improved Droop Control Method for DC Microgrids Based on Low Bandwidth Communication with DC Bus Voltage Restoration and Enhanced Current Sharing Accuracy. IEEE Trans. Power Electron. 2014, 29, 1800-1812. [CrossRef]

(C) 2019 by the authors. Licensee MDPI, Basel, Switzerland. This article is an open access article distributed under the terms and conditions of the Creative Commons Attribution (CC BY) license (http://creativecommons.org/licenses/by/4.0/). 